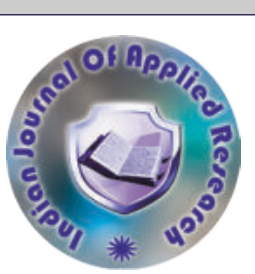

\title{
Cadiology \\ ACHILLES TENDINOUS XANTHOMAS WITH NEUROLOGICAL ABNORMALITIES IN CASE OF PRESENILE CATARACT: A CLUE TO CEREBROTENDINOUS XANTHOMATOSIS
}

\begin{tabular}{ll}
\hline $\begin{array}{l}\text { Dr Anamika } \\
\text { Meena* }\end{array}$ & $\begin{array}{l}\text { MBBS MD, All India Institute of Medical Sciences; Patna, Bihar, India. } \\
\text { *Corresponding Author }\end{array}$ \\
\hline $\begin{array}{l}\text { Dr Pranav } \\
\text { Santhalia }\end{array}$ & MBBS MD, All India Institute of Medical Sciences; Patna, Bihar, India . \\
\hline Dr Gunjan Kumar & MBBS, MD, DM, All India Institute of Medical Sciences; Patna, Bihar, India. \\
\hline $\begin{array}{l}\text { ABSTRACT A } 34 \text { years old female presented with gradual painless loss of vision in right eye and ataxia with past history of being } \\
\text { operated for cataract 12 years ago in left eye. On examination clinical examination, she had painless xanthomas of }\end{array}$ \\
$\begin{array}{l}\text { bilateral Achilles tendon, with ataxia and mild to moderate mental retardation and behavioral problems and further enquiring had history of } \\
\text { intractable diarrhea during infancy. Magnetic resonance imaging of brain showed bilateral and almost symmetrically increased T2 signal intensity } \\
\text { in the cerebellar and periventricular white matter, basal ganglia, dentate nuclei and brainstem along with cerebellar and bilateral frontal lobe } \\
\text { atrophy. }\end{array}$
\end{tabular}

\section{KEYWORDS :}

\section{INTRODUCTION}

Cerebrotendinous Xanthomatosis is a rare genetic (autosomal recessive inheritance) metabolic disease affecting the cholesterol excretion and leading to lipid deposition in different tissues, particularly tendons, central nervous system and eye lens (2). The onset of symptoms and signs in patients with CTX usually occurs in childhood or adolescence. Bilateral cataracts and diarrhea and neurologic abnormalities and tendon xanthomas are predominant symptoms in decreasing frequency (3). Neurologic symptoms include cerebellar and pyramidal dysfunction, cognitive impairment, dementia, epilepsy, and polyneuropathy (4). Other symptoms are premature atherosclerosis (mainly in coronary arteries) and osteoporosis (5). Xanthomas affecting the Achilles tendon bilaterally with a normal serum cholesterol level are unusual and are usually associated with CTX.

This case emphasizes the importance of suspecting CTX in the patient of presenile cataract with some neurological impairment and ataxia with bilateral Achilles tendon xanthomas.

\section{Case report}

A 34-year-old female patient with presenile cataract was admitted to the Department of Neurology with complaints of dyslexia and clinical examination revealed mental dysfunction, pyramidal signs, cerebellar dysfunction and dysarthria and large painless soft tissue swellings along the bilateral Achilles tendons (Fig. 1a, 1b), which on superficial ultrasound (Fig. 2a, 2b) suggested as xanthomas. On abdominal ultrasound Mild fatty changes in liver with cholelithiasis are seen. On 3 Tesla MRI (Siemens MAGNETOM) bilateral periventricular white matter (Fig. 3 a, 3b) and dentate nuclei (Fig. 3e) T2/FLAIR hyper intensities along with frontal lobe (Fig. 3 a, 3b) and cerebellar atrophy (Fig. 3d), hence tentative diagnosis of CTX was made.

Biochemical workup of patient revealed mildly elevated serum triglyceride levels with normal serum cholesterol. Plasma cholestanol, urine bile alcohol levels could not be evaluated due to unaffordability.

On clinical examination no cardiopulmonary abnormalities were seen and chest radiograph was normal. Nerve conduction velocity testing was also normal.
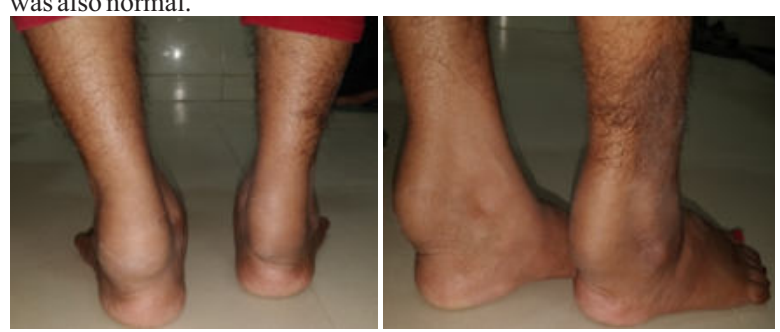

Fig. 1a, 1b showing bilateral Achilles tendon xanthomas

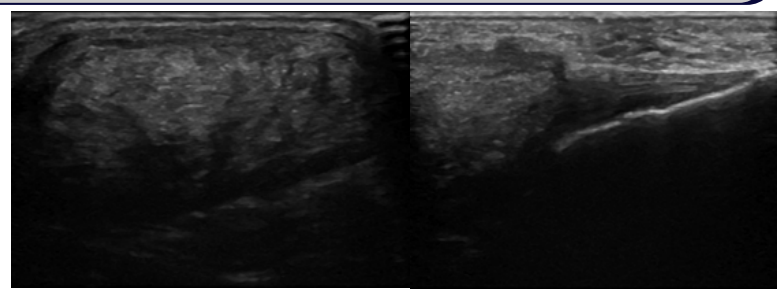

2a) USG transverse section shows globular thickening of Achilles tendon.

2b) USG longitudinal section shows fusiform thickening of Achilles tendon, just upto the level of insertion.

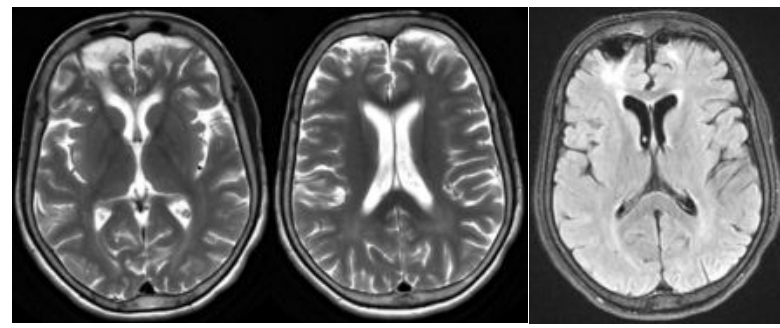

Fig. 3a, 3b) Axial T2 Weighted and ( Fig. 3c) FLAIR MRI image shows periventricular and right frontal lobe hyperintensity and Mild bilateral frontal lobe atrophy.

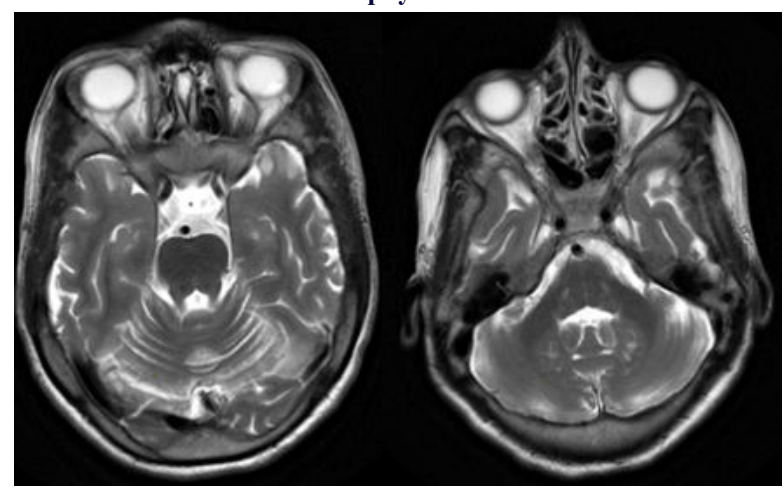

Fig.3d) Axial T2 Weighted MRI image shows cerebellar atrophy Fig 3e) Axial T2 Weighted MRI image shows subtle hyperintensity of both dendate nuclei

\section{DISCUSSION}

Earliest reports of CTX were made by Bogaert's in 1937 followed by few hundred cases worldwide (6). There are no consensus data on the prevalence of CTX, the estimated rate being $<5 / 100000$ worldwide. 
The clinical signs seen in patients affected by the disease are caused by storage of cholestanol in various tissues. Predilection sites for cholestanol storage are the brain and posterior fossa, eyes and the tendons. Elevation of plasma cholestanol levels is due to deficiency of a 26-hydroxylase mitochondrial enzyme which is involved in the metabolic breakdown of cholesterol.

CTX might generally being observed in the childhood with bilateral ocular involvement (cataract), chronic diarrhea and atherosclerosis, followed by neurological involvement and tendon xanthomas, the latter two findings being rarely observed before the age of twenty(6). Neurological manifestations include retardation of the neuropsychomotor development, epilepsy, cerebellar ataxia, spastic paraparesis, behavior disorders, dementia and polyneuropathy (7). Our patient also had most of similar clinical findings.

The most frequent neuroimaging findings in patients with CTX are nonspecific and include cerebral and cerebellar atrophy and periventricular white matter lesions. More characteristic lesions are seen in the basal ganglia, cerebral peduncles, and dentate nuclei (8). Subtle hyperintensity of the bilateral dentate nuclei and surrounding deep cerebellar white matter can be seen, as in our patient (Fig 5). $3 \mathrm{~T}$ MRI is potentially better as it substantially increase in signal intensity provided by higher magnetic field which eventually helps in early picking up of subtle hyper intensities in dentate nuclei and deep cerebellar white matter.

CTX is managed conservatively with administration of deoxycholic acid either alone or in combination with HMG-CoA reductase inhibitor which has shown to reduce the progression of disease. Hence our patient was started on ursodeoxycholic acid and HMG-CoA reductase inhibitors and she has put on regular follow up; however she was lost to follow up and no follow up clinical assessment and neuroimaging or neurophysiological assessment was done

Disorders associated with tendon xanthomas, such as sitosterolemia, hypercholesterolemia, and type IIa hyperlipidemia. On imaging there is no brain involvement and abnormal lipid profile on biochemistry evaluation. Clinically, CTX resembles Marinesco-Sjögren syndrome which is a triad of ataxia, mental retardation and congenital cataract. The presence of tendon xanthomas helps differentiate CTX from this condition and such differential is crucial as CTX is treatable if diagnosed early. Similarly bilateral dentate nuclei T2 hyper intensities can be seen in metronidazole toxicity and acute decompensated maple syrup urine disease however these patients clinically presents with acute encephalopathy in emergency settings. Langerhans histiocytosis also shows similar appearance although clinical history and Achilles tendon xanthomas will be lacking in such patients.

In summary, CTX is a rare genetic disorder with specific clinical, biochemical and radiological findings and potentially treatable with appropriate replacement therapy and hence early diagnosis is crucial before neurological deterioration starts. The imaging findings are $\mathrm{T} 2 \mathrm{~W}$ hyper-intensities in the Dentate nucleus, substantia nigra, globus pallidus and the presence of tendon xanthomas which are classic and should not be missed by the radiologist in appropriate clinical settings. Individual clinical, biochemical and radiological manifestations have multiple differentials but linking the multi-system findings suggests the diagnosis of CTX.

\section{Abbreviations:}

CTX: Cerebrotendinous xanthomatosis

CYP7A1: Cholesterol 7 $\alpha$-hydroxylase

CYP27A1 : Sterol 27-hydroxylase

3 T MRI:3 Tesla Meganatic Resonance Imaging

\section{REFERNCES:}

1. Lange MC, Zétola VF, Teive HAG, et al. Cerebrotendinous xanthomatosis: report of two Brazilian brothers. Arq Neuropsiquiatr. 2004;62:1085-9.

2. Wolthers Bg, Volmer M, Van Der Molen Jc, et al: Diagnosis of cerebrotendinous xanthomatosis (CTX) and effect of chenodeoxycholic acid therapy by analysis of urine, using capillary gas chromatography. Clin Chim Acta 131:53,1983.

3. Cruysberg Jr, Wevers Ra, Van Engelen Bg, et al: Ocular and systemic manifestations of Cruysberg Jr, Wevers Ra, Van Engelen Bg, et al: Ocular and system
cerebrotendinous xanthomatosis. Am J Ophthalmol 120:597, 1995.

4. Katz Da, Scheinberg L, Horoupian Ds, et al: Peripheral neuropathy in cerebrotendinous xanthomatosis. Arch Neurol 42: 1008, 1985.

5. Berginer Vm, Shany S, Alkalay D: Osteoporosis and increased bone fractures in cerebrotendinous xanthomatosis. Metabolism 42: 69, 1993.

6. Gallus GN, Dotti MT, Federico A (2006) Clinical and molecular diagnosis of cerebrotendinous xanthomatosis with a review of the mutations in the CYP27A1 gene. Neurol Sci 27: 143-149.

7. Sodhi HS, Agnish ND, Khudchedkar BJ (1973) Cholestanol biosynthesis in
8. Barkhof F, Verrips A, Wesseling P, et al. Cerebrotendinous xanthomatosis: the spectrum of imaging findings and the correlation with neuropathologic findings. Radiology 2000 ; 217(3):869-876. 